\title{
Perspectives
}

\section{Semen analysis in 21st century medicine: the need for sperm function testing}

\author{
Dolores J. Lamb
}

Scott Department of Urology and the Department of Molecular and Cellular Biology, Baylor College of Medicine, Houston, TX 77030, USA

\begin{abstract}
Sperm function testing, once commonly performed for the infertile couple before employing assisted reproductive technology (ART), has fallen out of favour in many reproductive medicine centers throughout the world. Indeed, the most recent addition of the 'World Health Organisation (WHO) Laboratory Manual for the Examination and Processing of Human Semen' now groups many of these procedures into a section termed Research Procedures. In large part, this reflects the current clinical practice of bypassing the in-depth evaluation of the male partner, while assuming that if a spermatozoon can be found for intracytoplasmic sperm injection (ICSI), it must be a healthy cell capable of achieving fertilization. Nevertheless, sperm function testing can provide valuable clinical insights into defects causing male infertility. Admittedly, in some cases, functional sperm deficiencies can be overcome using an ART. In other cases, couples will be empowered by the knowledge of the cause of their infertility, and for some couples, perhaps even the likelihood of ICSI success (relative to the spermatozoa). The knowledge allows them to make truly informed reproductive decisions, including (perhaps) the decision to seek donor insemination, to adopt or to remain childless. Knowledge of the cause of their infertility may provide closure for couples and a sense of confidence regarding their choice of reproductive treatment.
\end{abstract}

Asian Journal of Andrology (2010) 12: 64-70. doi: 10.1038/aja.2009.4

Keywords: semen analysis, sperm function tests

\section{Introduction}

With the advent of intracytoplasmic sperm injection (ICSI) [1], the evaluation and treatment of the infertile male radically changed. Just a single spermatozoon was required, motility was not necessary, and the normal biological processes of sperm capacitation, the acrosome reaction (AR), cumulus penetration, zona and ova binding, and penetration did not necessarily occur before fertilization. Although first used with ejaculated spermatozoa, in-

Correspondence to: Dr Dolores J. Lamb, Scott Department of Urology and the Department of Molecular and Cellular Biology, Baylor College of Medicine, One Baylor Plaza RM N730, Houston, TX 77030, USA.

Fax: +1-713-798-5577

Received: 6 November 2008

Accepted: 4 January 2009
E-mail: dlamb@bcm.tmc.edu Revised: 10 November 2008 vestigators soon realized that the spermatozoa retrieved by microsurgical epididymal spermatozoa aspiration, or even later, directly from the testis (testicular sperm extraction), could be used together with ICSI-in vitro fertilization (IVF) for men with non-obstructive azoospermia-even those men diagnosed with a Sertoli-cell-only pathology because of the presence of rare foci of spermatogenesis. As this assisted reproductive technology (ART), used in combination with IVF, overcame many of the deficiencies of sperm count, motility, morphology and function, the evaluation of the infertile male was frequently bypassed prior to ART, with the exception of a semen analysis. Indeed, in the most recent edition of the World Health Organization (WHO) Laboratory Manual for the Examination and Processing of Human Semen, although the more routine semen analysis procedures are described in detail, sperm function tests are now grouped into a chapter termed Research Procedures. In this commentary on the new Labora- 
tory Manual for the Examination and Processing of Human Semen, I will argue that there is still a place for sperm function testing in the state-of-the-art evaluation of the infertile male. I will describe several of the specialized functional andrology tests developed over the years and highlight the advantages and disadvantages of including this testing for the infertile couple in the era of ICSI. I will present evidence that the use of advanced sperm testing provides important insights into the abnormalities of spermatozoa that affect fertilization and that this knowledge is critical to a proper clinical evaluation of the infertile couple.

Routine semen analysis provides useful information concerning sperm production by the testis, sperm motility and viability, the patency of the male genital tract, the secretions of the accessory organs, as well as ejaculation and emission. Although the information revealed by this assay is obviously useful for the initial evaluation of the infertile male, it is not a test of fertility, and it provides no insights into the functional potential of the spermatozoon to fertilize an ovum or to undergo the subsequent maturation processes that are required to achieve fertilization. Indeed, the semen analysis result alone cannot distinguish the fertile from the infertile population [2,3], unless the man is azoospermic. Semen analysis also plays an important role in the assessment of effects of environmental toxicants or drug studies (for example, References $[4,5]$ ).

Accordingly, tests have been developed to define sperm function abnormalities in the infertile male. In the broadest sense, sperm function may simply be thought of as the ability of one spermatozoon to deliver the correct complement of chromosomes to an ovum. To do this, spermatozoa must be produced in sufficient numbers, and exhibit normal motility and shape (and these parameters generally are assessed in routine semen analysis). In addition, spermatozoa must be capable of penetrating and passing through the cervical mucus, and through the uterus to the ampullae of the oviducts, in addition to undergoing capacitation, AR, binding and penetration of the zona pellucida, and ultimately the ovum. Once the spermatozoon penetrates the ovum, it must then undergo nuclear decondensation to deliver the appropriate haploid chromosome complement; it then undergoes additional, but poorly understood, events required for fertilization and early embryonic development. Defects in any of these complex events can result in male infertility. Over the last 25 years, tests have been developed to identify abnormalities in these processes. These tests can have applications in evaluations of potential toxins, as well as in clinical trials for drug testing. However, the clinical use of these tests decreased significantly with the onset of ICSI, despite the fact that evaluation prior to treatment could prevent over treatment with the most advanced and costly technology, as well as unexpected IVF failure for men with normal se- men parameters (as measured by the semen analysis), but with unrecognized functional sperm deficiencies. With ICSI, many physicians treating the infertile couple no longer cared to determine the source of the infertility. If a spermatozoon could be found, there was the potential to fertilize an ovum, regardless of the functional deficiency. This fact is reflected in the current version of the WHO Manual for the Examination and Processing of Human Semen. A discussion of these tests and their relevance to the evaluation of the infertile male in the era of ICSI follows.

\section{Tests of sperm capacitation}

Capacitation refers to a collection of changes in sperm function that occur generally in the female genital tract. There is a change in the membrane permeability to calcium ions, which induce a hyper motility of the sperm, a process that is thought to aid in penetration of the cumulus and zona pellucida. Thus far, the assays developed to directly assess capacitation defects have only limited utility.

One of these tests, the AR test, measures the ability of the acrosome, essentially a modified Golgi apparatus limited by the inner and outer acrosomal membranes and located beneath the sperm plasma membrane, to release hydrolases (with acrosin being the predominant enzyme). This reaction subsequently allows the binding and penetration of the zona pellucida by the sperm cell. The AR occurs when the sperm plasma membrane and the outer acrosomal membrane fuse, an event that requires prior capacitation. Biochemical agents, such as calcium ionophore A23187 and progesterone, can induce the AR in vitro [6-8], and treatment of spermatozoa with these agents allows an assessment of the maximal potential induction of AR. The test methodologies vary and include several stains and antibodies to specific acrosomal components $[9,10]$. Yet studies have not compellingly shown that the AR assay results predict the likelihood of successful IVF, although some papers have reported a positive correlation [8]. With the development of IVF-ICSI, however, this AR assay is rarely used. The AR assay can definitively identify globozoospermia, but this defect is already easily identified when the strict (now routine) morphology assessment is performed. Electron microscopy remains the gold standard for globozoospermia (but this is rarely performed in the andrology laboratory). Nevertheless, for most clinical situations, the AR test is probably not required as the information needed about globozoospermia can be obtained by the morphological assessment of the spermatozoa. Whether quantitative measurement of acrosin is useful in the evaluation of the infertile male remains controversial. Despite several reports stressing the usefulness of acrosin measurements [11-13], the test is not widely performed, and correlation with fertilization outcomes are difficult. 
Rigorous, highly controlled, large-scale studies with appropriate positive and negative controls are therefore needed in this regard.

\section{Tests of hemizona and zona-pellucida binding}

Sperm binding and penetration of the zona pellucida are critically important to fertility. Embryologists noted that in couples with fertilization failure in IVF, spermatozoa sometimes failed to bind to the zona pellucida [14]. Overstreet and Hembree [15] first reported on the penetration of human zona pellucida by spermatozoa in 1976; 12 years later, Burkman et al. [16] developed a hemizona assay, designed to predict fertilization (or at least spermzonae interaction). However, the test was difficult for the average andrology or IVF laboratory to perform. Zona (either fresh or preserved) had to be collected from excess ova or ova that had failed to fertilize in IVF; the ova were then bisected, and matching halves had to be incubated with both fertile donor (control) and patient spermatozoa [16]. As micromanipulation skills were required (which limited broad use in the andrology laboratory), improvements were made to the assay, including the addition of vital dyes that stained spermatozoa fluorescent red or green. The differentially dyed donor and patient spermatozoa were then mixed with the zona for competitive binding [14]. Inherent in these assays is the caveat that the ova/ zona may not be normal because they were predominantly collected from failed IVF procedures. Whereas the donor controls (for either hemizona or vital dye methods) could provide indications of a zona deficiency, the interpretation and reproducibility of the test remained challenging for most laboratories. This test is no longer widely offered, although it does provide useful information about functional defects in sperm-zona interaction. Future development of a synthetic biomaterial that could provide a standard matrix to assess sperm-zona binding defects in a controlled, standardized and reproducible manner would be helpful in the evaluation of this functional sperm defect.

Synthetic substrates, such as hyaluronic acid beads, have been developed for a different sperm-binding application. Investigators wished to use the sperm-binding activity to select the optimal sperm for IVF-ICSI, with poorer quality or immature sperm binding to the hyaluronic acid [17-19]. While this work is ongoing, such tests of sperm zona binding, when properly utilized, could help select patients for IVF-ICSI by predicting the couples who would fail to fertilize in IVF, owing to a failure in this step of reproduction. If binding to hyaluronic acid could aid in selection of superior sperm for ICSI, this approach would represent a significant advance for the field.

\section{Sperm penetration assay or sperm capacitation}

\section{index or zona-free hamster oocyte penetration assay}

In 1976, Yanagamachi et al. [20] made the remarkable observation that after removal of their zona pellucida, the hamster eggs are promiscuous - they allow spermatozoa from several species (including humans) to penetrate. In the hamster, the block to fertilization by heterologous sperm acts at the level of zona pellucida. Yanagamachi et al. [20] developed the sperm penetration assay (SPA) using denuded hamster ova. The bioassay was designed to test sperm capacitation, as well as their ability to fuse with and penetrate the egg membrane and to undergo the initial steps of sperm head decondensation in a manner similar to that occurring during the initial events of fertilization. The test development correlated with the early development of IVF, and provided some predictive information regarding the potential for ART success.

Several modifications to this test were developed, but its predictive value was controversial. Some laboratories reported a high correlation with IVF outcome and a low false-positive rate $[21,22]$, whereas others reported an unacceptable level of false negatives, in part, due to differences in the rate of sperm capacitation between the protocols that influenced fertilization potential [23]. One reason for the high rate of false-negative predictive results was that after a failed SPA test, the IVF procedure used (the methodology differed markedly from the SPA) would be modified. Higher numbers of sperm would be added to the ova, and washing and sperm preparation techniques would be altered in an attempt to improve IVF outcome, because the ultimate goal was to achieve pregnancy. It was thus inevitable that such procedural variations contributed to the high false-negative rate.

Our laboratory then developed the 'optimized SPA', which is also called the sperm capacitation index or SCI $[24,25]$. The methodological changes to the assay resulted in polyspermy for normal fertile males (with the lower limit of normal being five spermatozoa per ovum), thus improving sensitivity while maintaining the specificity and positive predictive value to predict IVF success (fertilization). The test relied on the principles of clinical chemistry for assay design, definition of the normal range, and used both frozen controls of pooled semen samples and high- and low-penetrating sperm from fresh donors as standards. A positive score on this test was highly predictive of a positive outcome (fertilization) in IVF [24, 25], with the caveat that it could not identify defects in spermzona interaction. In theory, this test could identify couples with a high likelihood of success in IVF alone, as opposed to those requiring ICSI. The test is no longer commonly ordered today despite its potential to identify men with normal semen parameters, but who have functionally deficient spermatozoa, which are thus destined for fertilization 
failure in routine IVF.

Notably, a modification of the optimized SPA can predict fertilization outcome with IVF-ICSI. Gvakharia et al. [26], using a variation of the SPA, successfully predicted those men whose spermatozoa failed to decondense and fertilize after ICSI. Fertilization failure with ICSI was once considered to be a technical problem with the sperm injection itself; however, this paper identified men with a functional defect in their spermatozoa that prevented sperm head decondensation. Nevertheless, despite our ability to predict those severe male factor men whose spermatozoa will fail to fertilize eggs by ICSI, the hamster ICSI-SPA test is rarely ordered. With the knowledge gained from this test result, male factor couples with this sperm function defect would be saved the significant emotional and financial cost of a failed ICSI cycle, and could be counselled to seek an alternative path to parenthood.

\section{Tests of sperm DNA damage}

Whether or not to perform an assessment of sperm DNA damage during evaluation of the infertile male has been an area of controversy over the years. The causes of this DNA damage are largely unknown, although there is evidence to suggest that genetic defects may underlie some sperm DNA damage (reviewed in [27-29]). A variety of different tests are available: the acridine orange staining test, the sperm chromatin structural assay, terminal deoxynucleotidyl transferase-mediated nick end labelling and the Comet assay. Although each test measures DNA damage differently, all generally correlate well with each other (with the exception of the acridine orange staining test) and they also generally inversely correlate with sperm concentration [30]. In general, significant DNA damage is rarely found in a proven fertile male, and the incidence of DNA damage is higher in infertile men. Although most investigators would intuitively agree that DNA damage is probably detrimental, the clinical impact of DNA damage has been more challenging to discern, perhaps because the correct functional end points have not been identified. Recent studies suggest that sperm DNA damage levels can predict success using ART [31] and they may also predict the likelihood of recurrent pregnancy loss [32]. Zini et al. [33] suggested that sperm DNA damage may provide a useful biomarker of correction of detrimental fertility impairing conditions, such as varicocele. The incidence of DNA damage was lower in testicular spermatozoa compared with ejaculated spermatozoa; accordingly, ICSI with testicular spermatozoa might be advantageous to infertile men with high levels of DNA damage in their spermatozoa [34]. Additional highly controlled studies are required to define the functional significance of the DNA damage observed, the molecular basis for the damage and methods to prevent or ameliorate damage. Despite this relatively poor understanding of the nature and significance of the DNA damage observed, the presence of DNA damage is associated with male infertility. Although correlation with fertility outcomes [29] is not always obvious, one can distinguish fertile men from a sub-population of infertile men who exhibit significantly increased rates of sperm DNA damage. In essence, the presence of DNA damage may not provide a straightforward prediction of fertility potential. Nevertheless, DNA damage would not be detected in a routine semen analysis, making this test an important part of the evaluation of the infertile male.

\section{Assessment of reactive oxygen species}

Oxidative stress presents a major challenge in many aspects of medicine, pharmacology and cellular function. The generation of free radicals is a normal by-product of cellular metabolism. During oxidative metabolism, oxygen is enzymatically converted to produce energy. Coordinately, free radicals, as well as oxygen ions and peroxides, are generated. Naturally occurring antioxidants are present to protect cells, but cellular damage can occur when the normal homeostatic mechanisms are unbalanced. Reactive oxygen species (ROS) have been detected in the semen of some infertile men (reviewed in [35]). Indeed, the first report of a cell producing free radicals was by McLeod [36], who reported that incubation of spermatozoa under conditions of high oxygen tension leads to immotility. The addition of catalase, an antioxidant, preserved motility, thereby leading McLeod to speculate that spermatozoa produced hydrogen peroxide [36]. Another source of ROS is the neutrophil, the predominant leukocyte type in semen [37, 38]. However, a firm correlation between seminal white blood cell concentrations and ROS has been difficult to define. Other sources of free radicals are iatrogenic, lifestyle and environmental factors, local and systemic infection, and autoimmune, inflammatory, testicular and chronic disease (reviewed in [35]). ROS can damage the sperm membrane, with a detrimental effect on motility, and they may also damage the sperm DNA. Changes in lifestyle and exposures, supplementation with vitamins and antioxidants, and treatment of infection and inflammation may aid in the management of oxidative stress-related infertility [35]. Improvements in laboratory processing of semen or even surgical intervention may reduce damage from oxidative stress. Importantly, despite improvements in sperm quality with anti-oxidant treatment and improved semen processing techniques, improvement in pregnancy rates has not been as obvious. Research in the area of oxidative stress and male infertility is clearly needed. There is also a significant need for research that shows the efficacy of treatments in current clinical practice, such as admini- 
stration of antioxidants (with clinical trials that include placebo controls) to patients to prevent or ameliorate ROS generation, as some treatments may be futile depending upon the source of the ROS. Nevertheless, it is important to know whether ROS are present in semen, as antioxidant treatments are relatively simple to prescribe and generally well tolerated. With these treatments, together with lifestyle modifications that avoid toxins, improvement in sperm quality is generally expected; these treatments may even allow for natural conception for some couples.

\section{Chromosomal aneuploidy in sperm}

The most recent version of the WHO Laboratory Manual for the Examination and Processing of Human Semen does not include tests for the assessment of the chromosome complement of spermatozoa in either the routine semen testing or research chapters of the manual. This is surprising, as delivery of the proper chromosome complement by the spermatozoon to the ovum is arguably the most important function of sperm. Fluorescence in situ hybridization provides a method to test for sperm chromosomal aneuploidy. Aneuploidy, which is abnormality in the number of whole chromosomes in a cell, occurs as a result of a meiotic defect during spermatogenesis. As a result, a spermatozoon that is disomic or nullisomic for a particular chromosome will develop. Fertilization with this type of abnormal sperm cell results in monosomic or trisomic embryos, the majority of which are incompatible with a viable birth. Some of these aneuploidies result in an offspring with Down, Klinefelter, Patu, Edwards or Turner syndromes. Importantly, the spermatozoa of infertile men exhibit a 10-fold higher incidence of chromosome aneuploidies (testing for chromosomes X, Y, 13, 18, 21those compatible with a live birth) compared with fertile men. The increased incidence increases as the standard semen parameters worsen [39]. This sperm aneuploidy is present despite a normal somatic karyotype. Retrospective studies support the idea that an elevated rate of aneuploidy in spermatozoa is linked to the birth of a child (or conceptions) with aneuploidy [40-45]. Similarly, prospective studies show that men with elevated sperm aneuploidy are at increased risk of producing aneuploid embryos, IVF failure or aneuploid conceptuses [46-48]. Recurrent pregnancy loss is also associated with elevated sperm aneuploidy [49]. For couples with recurrent pregnancy loss, knowledge of increased sperm aneuploidy, together with genetic counseling, allows them to make informed reproductive decisions; their options include remaining childless, using donor sperm, using preimplantation genetic diagnosis together with ICSI-IVF, or to continue their efforts to achieve a naturally conceived or ICSI-IVFmediated pregnancy.

\section{Final comments}

I am often asked why the evaluation of the infertile male requires advanced, specialized andrology assessment of parameters, such as genetics or sperm function, if all that is needed is a single spermatozoon to achieve a pregnancy with ICSI (the logic being that if a fertilization can occur, a healthy baby is likely to have been conceived). Yet, we know this logic is flawed, as even healthy fertile couples may conceive a child with significant genetic or birth defects. Certainly, some of the tests of sperm function described above can provide important information to infertile couples to aid in their reproductive decision making. In some cases, sperm function testing may indicate that less expensive technologies may help a couple seeking to conceive a child; ICSI-IVF may not always be required. In other cases, such as men who fail the hamster ICSI-SPA test, donor spermatozoa or other options must be considered. The real strength of sperm function testing lies in its ability to identify men with normal semen parameters but who have functionally deficient spermatozoa that will fail to fertilize in routine IVF. These functional deficiencies will never be observed on a routine semen analysis. As they provide a unique insight into sperm physiology, sperm function tests may also aid in the design of improved treatment that are provided to infertile couples.

With the molecular revolution that has occurred over the last 15 years, our understanding of the genetic defects that underlie defects in sperm function, as well as the processes of spermatogenesis and fertilization, has improved. Defects that impair sperm function, revealed by the tests described in this paper, frequently have a genetic basis (reviewed in [27, 28]); some affect basic cellular processes that impact the individual in ways beyond their ability to reproduce. Although diagnostic tests are not currently available for each gene, other tests, such as those developed to assess sperm aneuploidy, provide important information to couples planning to use ART. The use of these tests to identify couples absolutely requiring ICSIIVF to achieve a pregnancy, or even those who are likely to fail to achieve a pregnancy with ICSI-IVF, will allow physicians to provide a cost-efficient treatment plan for each infertile male factor couple. Most importantly, an infertile couple undertakes a tortuous journey in their quest to become parents, a journey that is fraught with huge financial and emotional costs that are rarely considered by the medical community. Sperm function tests, such as those described above, can provide increased knowledge of the causes of infertility, frequently bringing closure to this quest. The results of such testing may allow for the use of more cost-efficient treatment or even provide the basis for a couple to cease treatment. Ultimately, it is the 
couple's decision to seek to achieve a pregnancy; infertile couples, however, deserve the right to decide whether this is a realistic goal with a reasonable likelihood of success. Thus, sperm function testing, as well as other genetic and andrology tests now available, allow couples to make a more informed decision.

\section{Acknowledgment}

I thank my colleague, Dr Joseph Alukal, for helpful comments regarding this manuscript. Reproductive biology and cancer research in the Lamb laboratory is supported by US National Institutes of Health Grants P01 HD36289, R01 DK078121, U54 HD07495, T32 DK00763 and K12 DK083014, by the US Department Of Defense, US Army Materiel Command PC061154.

\section{References}

1 Palermo G, Joris H, Devroey P, Van Steirteghem AC. Preg nancies after intracytoplasmic injection of single spermatozoon into an oocyte. Lancet 1992; 340: 17-8.

2 Guzick DS, Overstreet JW, Factor-Litvak P, Brazil CK, Nakajima ST, et al. Sperm morphology, motility, and concentration in fertile and infertile men. N Engl J Med 2001; 345: 1388-93.

3 Smith KD, Rodriguez-Rigau LJ, Steinberger E. Relation between indices of semen analysis and pregnancy rate in infertile couples. Fertil Steril 1977; 28: 1314-9.

4 Rubes J, Selevan SG, Evenson DP, Zudova D, Vozdova M, et al. Episodic air pollution is associated with increased DNA fragmentation in human sperm without other changes in semen quality. Hum Reprod 2005; 20: 2776-83.

5 Rubes J, Selevan SG, Sram RJ, Evenson DP, Perreault SD. GSTM1 genotype influences the susceptibility of men to sperm DNA damage associated with exposure to air pollution. Mutat Res 2007; 625: 20-8.

6 Perry RL, Naeeni M, Barratt CL, Warren MA, Cooke ID. A time course study of capacitation and the acrosome reaction in human spermatozoa using a revised chlortetracycline pattern classification. Fertil Steril 1995; 64: 150-9.

7 Oehninger S, Blackmore P, Morshedi M, Sueldo C, Acosta AA, et al. Defective calcium influx and acrosome reaction (spontaneous and progesterone-induced) in spermatozoa of infertile men with severe teratozoospermia. Fertil Steril 1994; 61: 34954.

8 Yovich JM, Edirisinghe WR, Yovich JL. Use of the acrosome reaction to ionophore challenge test in managing patients in an assisted reproduction program: a prospective, double-blind, randomized controlled study. Fertil Steril 1994; 61: 902-10.

9 Kallajoki M, Virtanen I, Suominen J. The fate of acrosomal staining during the acrosome reaction of human spermatozoa as revealed by a monoclonal antibody and PNA-lectin. Int J Androl 1986; 9: 181-94.

10 Sanchez R, Toepfer-Petersen E, Aitken RJ, Schill WB. A new method for evaluation of the acrosome reaction in viable human spermatozoa. Andrologia 1991; 23: 197-203.

11 Chaudhury K, Das T, Chakravarty B, Bhattacharyya AK. Acrosin activity as a potential marker for sperm membrane charac- teristics in unexplained male infertility. Fertil Steril 2005; 83: 104-9.

12 Langlois MR, Oorlynck L, Vandekerckhove F, Criel A, Bernard $\mathrm{D}$, et al. Discrepancy between sperm acrosin activity and sperm morphology: significance for fertilization in vitro. Clin Chim Acta 2005; 351: 121-9.

13 Nasr-Esfahani MH, Razavi S, Tavalaee M. Failed fertilization after ICSI and spermiogenic defects. Fertil Steril 2008; 89: 892-8.

14 Liu DY, Clarke GN, Lopata A, Johnston WI, Baker HW. A sperm-zona pellucida binding test and in vitro fertilization. Fertil Steril 1989; 52: 281-7.

15 Overstreet JW, Hembree WC. Penetration of the zona pellucida of nonliving human oocytes by human spermatozoa in vitro. Fertil Steril 1976; 27: 815-31.

16 Burkman LJ, Coddington CC, Franken DR, Krugen TF, Rosenwaks $\mathrm{Z}$, et al. The hemizona assay (HZA): development of a diagnostic test for the binding of human spermatozoa to the human hemizona pellucida to predict fertilization potential. Fertil Steril 1988; 49: 688-97.

17 Huszar G, Ozenci CC, Cayli S, Zavaczki Z, Hansch E, et al. Hyaluronic acid binding by human sperm indicates cellular maturity, viability, and unreacted acrosomal status. Fertil Steril 2003; 79 (Suppl 3): 1616-24.

18 Huszar G, Ozkavukcu S, Jakab A, Celik-Ozenci C, Sati GL, et al. Hyaluronic acid binding ability of human sperm reflects cellular maturity and fertilizing potential: selection of sperm for intracytoplasmic sperm injection. Curr Opin Obstet Gynecol 2006; 18 : $260-7$.

19 Huszar G, Jakab A, Sakkas D, Ozenci CC, Cayli S, et al. Fertility testing and ICSI sperm selection by hyaluronic acid binding: clinical and genetic aspects. Reprod Biomed Online 2007; 14: 650-63.

20 Yanagimachi R, Yanagimachi H, Rogers BJ. The use of zonafree animal ova as a test-system for the assessment of the fertilizing capacity of human spermatozoa. Biol Reprod 1976; 15 : 471-6.

21 Aitken RJ, Irvine DS, Wu FC. Prospective analysis of spermoocyte fusion and reactive oxygen species generation as criteria for the diagnosis of infertility. Am J Obstet Gynecol 1991; 164: $542-51$.

22 Margalioth EJ, Feinmesser M, Navot D, Mordel N, Bronson RA. The long-term predictive value of the zona-free hamster ova sperm penetration assay. Fertil Steril 1989; 52: 490-4.

23 Perreault SD, Rogers BJ. Capacitation pattern of human. spermatozoa. Fertil Steril 1982; 38: 258-60.

24 Johnson A, Bassham B, Lipshultz LI, Lamb DJ. A quality control system for the optimized sperm penetration assay. Fertil Steril 1995; 64: 832-7.

25 Smith RG, Johnson A, Lamb D, Lipshultz LI. Functional tests of spermatozoa. Sperm penetration assay. Urol Clin North Am 1987; 14: 451-8.

26 Gvakharia MO, Lipshultz LI, Lamb DJ. Human sperm microinjection into hamster oocytes: a new tool for training and evaluation of the technical proficiency of intracytoplasmic sperm injection. Fertil Steril 2000; 73: 395-401.

27 Matzuk MM, Lamb DJ. The biology of infertility: research advances and clinical challenges. Nat Med 2008; 14: 1197-213.

28 Matzuk MM, Lamb DJ. Genetic dissection of mammalian fertility pathways. Nat Cell Biol 2002; 4 (Suppl): S41-9.

29 Practice Committee of the American Society for Reproductive 
Medicine. The clinical utility of sperm DNA integrity testing. Fertil Steril 2006; 86 (5 Suppl): S35-7.

30 Chohan KR, Griffin JT, Lafromboise M, De Jonge CJ, Carrell DT. Comparison of chromatin assays for DNA fragmentation evaluation in human sperm. J Androl 2006; 27: 53-9.

31 Bungum M, Humaidan P, Axmon A, Spano M, Bungum L, et al. Sperm DNA integrity assessment in prediction of assisted reproduction technology outcome. Hum Reprod 2007; 22: 174-9.

32 Carrell DT, Liu L, Peterson CM, Jones KP, Hatasaka HH, et al. Sperm DNA fragmentation is increased in couples with unexplained recurrent pregnancy loss. Arch Androl 2003; 49: 49-55.

33 Zini A, Blumenfeld A, Libman J, Willis J. Beneficial effect of microsurgical varicocelectomy on human sperm DNA integrity. Hum Reprod 2005; 20: 1018-21.

34 Greco E, Scarselli F, Iacobelli M, Rienzi L, Ubaldi F, et al. Efficient treatment of infertility due to sperm DNA damage by ICSI with testicular spermatozoa. Hum Reprod 2005; 20: 226-30.

35 Tremellen K. Oxidative stress and male infertility-a clinical perspective. Hum Reprod Update 2008; 14: 243-58.

36 McLeod J. The role of oxygen in the metabolism and motility of spermatozoa. Am J Physiol 1943; 138: 512-8.

37 Aitken RJ, West K, Buckingham D. Leukocytic infiltration into the human ejaculate and its association with semen quality, oxidative stress, and sperm function. J Androl 1994; 15: 343-52.

38 Aitken RJ, Baker HW. Seminal leukocytes: passengers, terrorists or good samaritans? Hum Reprod 1995; 10: 1736-9.

39 Vegetti W, Van Assche E, Frias A, Verheyen G, Bianchi MM, et al. Correlation between semen parameters and sperm aneuploidy rates investigated by fluorescence in-situ hybridization in infertile men. Hum Reprod 2000; 15: 351-65.

40 Blanco J, Gabau E, Gomez D, Baena N, Guitart M, et al. Chromosome 21 disomy in the spermatozoa of the fathers of children with trisomy 21 , in a population with a high prevalence of Down syndrome: increased incidence in cases of paternal origin. Am J Hum Genet 1998; 63: 1067-72.

41 Martinez-Pasarell O, Nogues C, Bosch M, Egozcue J, Templado C. Analysis of sex chromosome aneuploidy in sperm from fathers of Turner syndrome patients. Hum Genet 1999; 104: 345-9.

42 Martinez-Pasarell O, Templado C, Vicens-Calvet E, Egozcue J, Nogues C. Paternal sex chromosome aneuploidy as a possible origin of Turner syndrome in monozygotic twins: case report. Hum Reprod 1999; 14: 2735-8.

43 Carrell DT, Wilcox AL, Udoff LC, Thorp C, Campbell B. Chromosome 15 aneuploidy in the sperm and conceptus of a sibling with variable familial expression of round-headed sperm syndrome. Fertil Steril 2001; 76: 1258-60.

44 Soares SR, Templado C, Blanco J, Egozcue J, Vidal F. Numerical chromosome abnormalities in the spermatozoa of the fathers of children with trisomy 21 of paternal origin: generalised tendency to meiotic non-disjunction. Hum Genet 2001; 108: 134-9.

45 Soares SR, Vidal F, Bosch M, Martinez-Pasarell O, Nogues C, et al. Acrocentric chromosome disomy is increased in spermatozoa from fathers of Turner syndrome patients. Hum Genet 2001; 108: 499-503.

46 Martin RH. Sperm chromosome analysis in a man heterozygous for a paracentric inversion of chromosome 7 (q11q22). Hum Genet 1986; 73: 97-100.

47 Nagvenkar P, Zaveri K, Hinduja I. Comparison of the sperm aneuploidy rate in severe oligozoospermic and oligozoospermic men and its relation to intracytoplasmic sperm injection outcome. Fertil Steril 2005; 84: 925-31.

48 Gianaroli L, Magli MC, Ferraretti AP. Sperm and blastomere aneuploidy detection in reproductive genetics and medicine. J Histochem Cytochem 2005; 53: 261-7.

49 Rubio C, Simon C, Blanco J, Vidal F, Minguez Y, et al. Implications of sperm chromosome abnormalities in recurrent miscarriage. J Assist Reprod Genet 1999; 16: 253-8. 